\title{
Evolution of Binaries Towards HMXBs and LMXBs
}

\author{
B. Kalomeni \\ University of Ege, Department of Astronomy \& Space Sciences, 35100, Bornova-İzmir, Turkey \\ Department of Physics, İzmir Institute of Technology, 35430, Urla-İzmir, Turkey
}

\begin{abstract}
In this study we investigate the evolution of binary systems towards low mass x-ray binaries and high mass x-ray binaries using Eggleton's binary star evolution code.
\end{abstract}

Keywords: Binary systems, HMXBs, LMXBs, stellar evolution

PACS: $97.80 . J p$

\section{INTRODUCTION}

X-ray binary systems consist of a mass transferring star and a compact star (a black hole, neutron star or possibly a white dwarf). The matter falling onto the compact star is the source of X-rays. X-ray binaries based on the mass of the donor star are classified as High Mass X-ray Binaries (HMXBs) and Low Mass X-ray Binaries (LMXBs). HMXBs form an important class of close binaries in the sense that they give the opportunity to study massive $\mathrm{OB}$ stars and the nature of compact object. For the formation of LMXBs different processes have been discussed in the literature (e.g. [1], [2], [3]).

\section{PRELIMINARY RESULTS}

To study the evolution of a single star and stars in a binary system generally stellar evolution codes have been used in the literature (see for instance [4]). We have used the binary evolution STARS code ([5], [6], [7], [8]) in which the evolution of both stars is considered. A variety of combinations are taken into consideration. After some runs, we saw the progenitor primaries with masses between $6.5 \mathrm{M}_{\odot}-14 \mathrm{M}_{\odot}$ can form neutron star LMXBs and HMXBs, depending the mass of the component star. The mass of the secondary and the orbital period are the crucial parameters to determine the fate of the massive star. To study the progenitor of LMXBs we have considered binaries with low initial masses $\left(\mathrm{M}_{2}<2 \mathrm{M}_{\odot}\right)$. In the case where the mass of the secondary is close to its primary a HMXB can be formed. During the evolution of the system the primary losses mass and the separation of the components and therefore the orbital period increases. If the initial orbital period is high the components apparently do not interact with eachother. The results show that for the mass ratios smaller than 1.5 HMXBs are formed while for $\mathrm{q}>10 \mathrm{LMXBs}$ are formed. Figures concerning the evolutionary path of the progenitor stars are depicted in Fig. 1 and Fig. 2 for a HMXB and for a LMXB, respectively. 

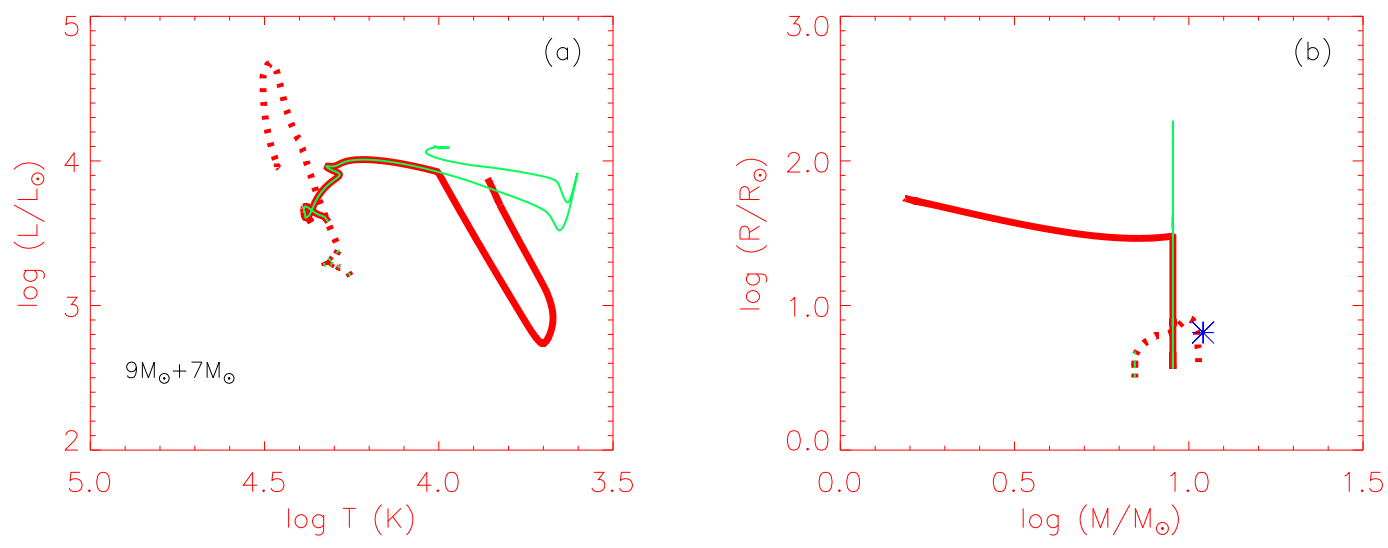

FIGURE 1. (a) Evolutionary track of a binary system with initial masses of $9 \mathrm{M}_{\odot}$ (solid red line) and $7 \mathrm{M}_{\odot}$ (dashed red line). The evolutionary tracks of single stars with initial masses of $9 \mathrm{M}_{\odot}$ (solid green line) and $7 \mathrm{M}_{\odot}$ (dashed green line) are also shown. (b) The M-R relation of the components. The blue star indicates the mass and radius of the component star in the HMXB system PSR J1740-3052. The mass and radius value of the component of PSR J1740-3052 obtained from [9].

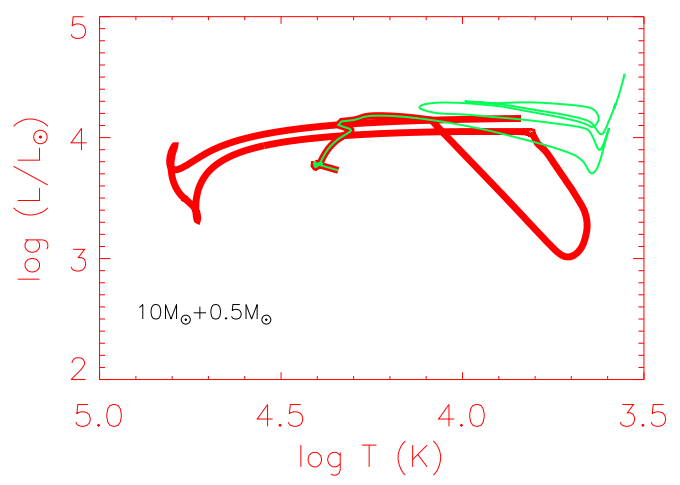

FIGURE 2. Evolutionary track of a star with initial mass of $10 \mathrm{M}_{\odot}$ (solid red line) in a binary system with a $0.5 \mathrm{M}_{\odot}$ component. The evolutionary tracks of single star with initial masses of $10 \mathrm{M}_{\odot}$ is shown with solid green line.

\section{REFERENCES}

1. V. Kalogera, and R. F. Webbink, ApJ 458, 301-311 (1996).

2. V. Kalogera, and R. F. Webbink, $A p J 493$, 351-367 (1998).

3. P. P. Eggleton, and F. Verbunt, MNRAS 220, 13P-18P (1986).

4. O. R. Pols, $A \& A$ 290, 119-128 (1994).

5. P. P. Eggleton, MNRAS 151, 351-364 (1971).

6. P. P. Eggleton, Private communication (2010).

7. O. R. Pols, C. A. Tout, P. P. Eggleton, and Z. Han, MNRAS 274, 964-974 (1995).

8. R. J. Stancliffe, and J. J. Eldridge, MNRAS 396, 1699-1708 (2009), 0904 . 0282.

9. I. H. Stairs, and et al., MNRAS 325, 979-988 (2001). 